\title{
1 Genomic hypervariability of phage Andromeda is unique among known
}

\section{2 dsDNA viruses}

3

4 Damian J. Magill ${ }^{1 \dagger}$, Leonid A. Kulakov ${ }^{1}$, Timofey A. Skvortsov ${ }^{2 *}$

5

$6{ }^{1}$ Queen's University Belfast, School of Biological Sciences,

719 Chlorine Gardens, Belfast BT9 5DL, Northern Ireland

${ }^{2}$ Queen's University Belfast, School of Pharmacy, Medical Biology Centre,

997 Lisburn Road, Belfast BT9 7BL, Northern Ireland

* Correspondence: Timofey A. Skvortsov, Queen's University Belfast, School of Pharmacy, 13 Medical Biology Centre, 97 Lisburn Road, Belfast, BT9 7BL, Northern Ireland; email:

${ }^{\dagger}$ Present address: DuPont (Danisco), Nutrition \& Health, Route de Buxières - CS 10010,

Keywords: bacteriophage; phage; genome hypervariability; quasispecies;

\section{Autographivirinae.}


Abstract

A new lytic bacteriophage Andromeda, specific to the economically important plant pathogen Pseudomonas syringae, was isolated and characterised. It belongs to the

43 Podoviridae family, Autographivirinae subfamily and possesses a linear dsDNA genome of 40,008 bp with four localised nicks. Crucially, Andromeda's genome has no less than 80 hypervariable sites (SNPs), which show genome wide distribution resulting in heterogenous populations of this phage reminiscent of those of RNA virus quasispecies. Andromeda has no nucleotide sequence homology to phage phiNFS, a member of phiKMVviruses, in which a similar phenomenon was discovered. We show that Andromeda and Andromeda-related phages form a group within the Autographivirinae, designated here as the "ExophiKMVviruses". The "ExophiKMVviruses" were revealed to

51 share conservation of gene order with core phiKMVviruses despite their sequence-based relationship to SP6-related phages. Our findings suggest that genomic hypervariability might be a feature that occurs among various Autographivirinae groups. 
55

56

\section{Introduction}

The phiKMVviruses constitute a ubiquitously distributed group of bacteriophages closely related to Teseptimaviruses and Zinderviruses. These three large groups and a number of smaller ones fall under the Autographivirinae subfamily, the major difference between them being the localisation of their single subunit RNA polymerase enzymes. With respect to phiKMVviruses in particular, the majority of phages infect Pseudomonas strains, with first representatives being described in 1980s [1]. phiKMVviruses are of interest for various reasons. Those infecting Pseudomonas aeruginosa exhibit a number of interesting features including the presence of four localised single-strand interruptions (nicks) associated with the consensus sequence 5'-CGACNNNNNCCTACTCCGG-3' [1, 2]. The more recent study revealed that at least some phiKMV phages exhibit hypervariability associated with specific genomic regions [3]. These hypervariable loci were found in genes associated with phage adsorption/host specificity and may therefore influence the evolutionary pathway of the phages [3].

This study describes the biological properties of a novel phage, Andromeda, which infects Pseudomonas syringae. The presence of a large number of hypervariable sites in the Andromeda's genome and its relationship to phiKMVviruses are investigated.

\section{Materials and Methods}

\section{Phage Isolation and Purification}

Bacteriophage Andromeda was isolated by enrichment of $100 \mathrm{ml}$ of water from River Lagan (Belfast, Northern Ireland) in cultures of Pseudomonas syringae pv syringae Van Hall followed by plating using the double agar overlay plaque assay [4]. Pure isolates of the phage were obtained by conducting three rounds of phage propagation from individual plaques. To obtain high titre preparations of the phage, cultures of $P$. syringae $\left(\mathrm{OD}_{600}=\right.$ 0.3 ) were inoculated at a $\mathrm{MOI}=0.01$. Chloroform was added immediately after culture lysis ( 4 hours) to maximise yield. Resistance of the phage particles to chloroform treatment was determined in separate experiments. The phage lysate was concentrated by PEG precipitation, and purified using $\mathrm{CsCl}$ density gradient centrifugation as described by Sambrook and Russell [4]. Bands were extracted and dialysis ensued with modified SM buffer (100 mM NaCl, 50 mM Tris, 10 mM, $8 \mathrm{mM} \mathrm{MgSO}_{4}, 10 \mathrm{mM} \mathrm{CaCl}_{2}$ ) using Amicon Ultra-15 100kDa filters (Millipore). Phage concentrates were treated with SDS and 
90 proteinase $\mathrm{K}$, after which phenol-chloroform DNA extraction followed by ethanol

91 precipitation was used to obtain phage DNA [4]. DNA was further purified twice prior to

92 sequencing using the MoBio DNA Purification Kit according to the manufacturer's

93 instructions.

94

\section{Electron Microscopy}

Carbon-coated formovar grids were subjected to hydrophilic treatment with poly-L-lysine for 5 min. Upon drying, $\mathrm{CsCl}$ purified and dialysed phage suspensions were deposited on the grids and successively stained with $2 \%$ uranyl acetate $(\mathrm{pH} 4.5)$. Samples were observed using a Phillips CM100 transmission electron microscope at $100 \mathrm{KeV}$.

\section{Determination of Adsorption Coefficient}

Determination of adsorption efficiency of phages was carried out using the method described in [5]. All adsorption assays were conducted in triplicate with final adsorption constant calculated from the mean of the replicate constants.

\section{One-step Growth Curves}

107 Determination of bacteriophage one-step growth curves was conducted according to the established protocol. Bacterial cultures were grown until $\mathrm{OD}_{600}=0.3\left(10^{8} \mathrm{CFU} / \mathrm{ml}\right)$ and inoculated with phage to give $\mathrm{MOI} \geq 1$. Adsorption was allowed for $5 \mathrm{~min}$ at $37^{\circ} \mathrm{C}$ followed by incubation with aeration at the same temperature. Duplicate $100 \mu$ l samples were taken every 5 minutes for 90 minutes. One of each samples were treated with $150 \mu$ of chloroform and both were plated on the lawns of $P$. syringae using the double agar overlay

113 plaque assay. Burst size was calculated by dividing the average of the values following the

114 growth phase of the curve (the step) by the average of the baseline values before the first

115 increase was observed in titre. The average of triplicates was then taken as the burst size.

116 Latent periods were taken as the point in the graph of the untreated phage extraction

117 whereby a rise in titre was observed, whereas the eclipse period was determined from the 118 chloroform treated curve.

\section{Visualisation of localised single-strand interruptions}

121 Detection of localised nicks were conducted as described earlier [2]. Phage DNA (3 $\mu \mathrm{g}$ )

122 underwent overnight ligation at $4^{\circ} \mathrm{C}$ in a final volume of $20 \mu \mathrm{l}$. $\mathrm{NaOH}$ was added to

123 reactions to a final concentration of $50 \mathrm{mM}$. This was also done for $1.8 \mu \mathrm{g}$ of unligated

124 DNA. Both samples were incubated for 5 min on ice and immediately analysed by gel 
electrophoresis $(0.9 \%$ agarose $)$.

\section{Library Preparation and Sequencing}

128 Sequencing libraries were prepared from $50 \mathrm{ng}$ of phage genomic DNA using the Nextera DNA Sample Preparation Kit (Illumina, USA) at the University of Cambridge Sequencing

130 Facility. A 1\% PhiX v3 library spike-in was used as a quality control for cluster generation

131 and sequencing. Sequencing of the resulting library was carried out from both ends (2x300

132 bp) with the 600-cycle MiSeq Reagent Kit v3 on MiSeq (Illumina, USA) and the adapters

133 trimmed from the resulting reads at the facility.

\section{Sequence Assembly and Bioinformatics}

136 Sequencing reads underwent initial quality checking using FastQC

137 (https://www.bioinformatics.babraham.ac.uk/projects/fastqc/) followed by stringent

138 trimming parameters. Reads with a Q-score < 20, containing N's, and with a final length <

139 40bp were discarded using Trimmomatic v0.32 [6]. Assembly was carried out using

140 Geneious R8 (Biomatters, New Zealand) and checked for potential misassembly by

141 comparison with output from SPAdes v3.7.0 [7]. Genome length contigs were input to the

142 scaffolding tool SSPACE to extend these in order to ensure the resolution of genome ends

143 [8]. In addition, the sequence obtained was utilised as a reference for read mapping using

144 bbmap v35.x (https://jgi.doe.gov/data-and-tools/bbtools/) and subsequent manual

145 inspection in IGV to check for potentially ambiguous regions [9]. ORFs were classified

146 using the intrinsic Artemis [10] ORF classification and GeneMark.hmm [11] followed by

147 manual inspection, ORF borders correction and verification of ribosomal binding sites.

148 Gene annotation was carried out using BLASTp, Delta-BLAST, HMMER, InterProScan

149 and HHpred [12-15]. tRNAs were classified using tRNA-scanSE and Aragorn [16, 17], and

150 rho-independent terminators were identified using Arnold [18] with an energy cutoff of -10

151 employed, following by manual inspection of the output from subsequent Mfold analysis

152 and adjustment as necessary [19]. To identify putative regulatory sequences, $100 \mathrm{bp}$

153 upstream of every ORF was extracted using an in-house script and analysed using both

154 neural network promoter prediction for prokaryotic sigma 70 promoters and Multiple EM for

155 Motif Elicitation to identify phage specific promoters [20]. Phage Andromeda assembled

156 genomic sequence was deposited to NCBI Genbank (Genbank accession number:

157 KX458241; RefSeq accession number: NC_031014). A reannotated genome of phage

158 Andromeda in Genbank format can be found in Supplementary File 1. 


\section{Phylogenetic analysis}

161 In order to taxonomically annotate the newly isolated phage and determine its

162 phylogenetic relationships with other bacteriopahges, two conserved Andromeda proteins,

163 the single subunit RNA polymerase (RNAP, YP_009279548.1) and the terminase large

164 subunit (TerL, YP_009279565.1), were used to generate phylogenetic trees. BLASTp

165 search with each of these proteins against Podoviridae available in RefSeq at the moment

166 of writing was conducted and only phage genomes containing copies of both TerL and

167 RNAP with high enough similarity (e-value $\leq 1 \mathrm{e}-5$; query coverage $\geq 75 \%$; percent identity

$168 \geq 25 \%$ ) to corresponding Andromeda's proteins were retained for subsequent analyses;

169147 complete phage genomes were selected in total (Supplementary Table 1). Alignments

170 of the amino acid sequences of RNAP and TerL were carried out in MAFFT using L-INS-I

171 settings [21], TrimAl was used to for automated alignment trimming [22]. The inference of

172 phylogenies was then carried out using IQ-Tree [23], which was allowed to compute the

173 optimal protein model to be used. Visualisation of the resulting tree file was subsequently

174 performed with the Interactive tree of life (IToL) [24]. The GGgenes package

175 (https://CRAN.R-project.org/package=gggenes) for R programming environment

176 (http://www.R-project.org/) was used to generate a series of genome maps.

178 Molecular Modelling

179 Molecular models were constructed from corresponding amino acid sequences using the I-

180 Tasser package [25] and visualised with Chimera [26]. Fidelity of models was assessed

181 using the validation tools of the Whatif server (https://swift.cmbi.umcn.nl/whatif/) and

182 checked for stereochemical clashes within Chimera. Ramachandran analysis was

183 subsequently carried out as an additional point of quality assessment. Superimposition

184 and quantitative similarity of protein structures was provided by the TM-Align algorithm

185 [27]. 


\section{Results and Discussion}

\section{Isolation and Biological Characteristics}

190 Bacteriophage Andromeda was isolated from the River Lagan as described in Materials

191 and Methods. Upon infection of Pseudomonas syringae, phage Andromeda yields large,

192 clear plaques of $\sim 3 \mathrm{~mm}$ in size. Electron microscopic analysis of Andromeda revealed a

193 virion morphology characteristic of Podoviridae (Fig. 1). Specifically, Andromeda was

194 found to possess a capsid of icosahedral symmetry with a length of $61 \mathrm{~nm}( \pm 1.5 \mathrm{~nm})$ and

195 width of $62 \mathrm{~nm}( \pm 1 \mathrm{~nm})$ and a detectable tail structure.

196 Analysis of the growth characteristics revealed an adsorption coefficient of $1.48 \times 10^{-9}$

$197 \mathrm{ml} \mathrm{min}{ }^{-1}$ (Fig. 2a), eclipse period of $20-25 \mathrm{~min}$, and latent period of $25-30 \mathrm{~min}$ (Fig. $2 \mathrm{~b}$ ).

198 Upon completion of its infection cycle, Andromeda yields on average 70 particles per cell.

\section{Genome Structure and Gene Regulation}

201 Phage DNA was sequenced as described in Materials and Methods to a per base average 202 coverage of 2149. The assembly and subsequent analysis were conducted and these 203 revealed a linear dsDNA genome of 40,008 bp (Supplementary File 1).

204 The GC content of the genome is $58.23 \%$ and using cumulative GC skew analysis, the 205 putative origin of replication was found to be at $3001 \mathrm{bp} .46$ ORFs were predicted within 206 the Andromeda genome resulting in a total coding potential of $95.1 \%$. Six ORFs utilise 207 TTG, and 1 utilises GTG as start codons with the rest beginning with ATG. All ORFs are encoded off the same strand. Analysis of the Andromeda genome by both the PhageTerm

209 [28] and Li's methods [29] revealed that this phage is terminally redundant and likely 210 utilises a headful mechanism of packaging similar to that of phage P1 (+ve strand analysis

$211(p=1.17 e-4)$, -ve strand analysis $(p=2.81 e-6))$. Based on nucleotide homology, the

212 closest relative to Andromeda is the $P$. tolaasii phage $\mathrm{Bf7}$ [30] with which it shares 83\%

213 identity across $85 \%$ of its genome (Fig. 3). Of the 46 ORFs, 25 were functionally assigned

214 and are arranged within a strict modular architecture. Eight ORFs encode products

215 associated with DNA replication, recombination, and repair. In particular, the presence of a

216 single subunit RNA polymerase of T3/T7 type was detected (Fig. 3; RNAP), which is

217 located downstream of the DNA polymerase (DNAP). This RNA polymerase is conserved

218 within Autographivirinae and exhibits similarity to that of phiNFS, particularly in the $\mathrm{N}$ - and

219 C- termini, the specificity loop, and the palm and finger domains. Indeed, molecular

220 modelling of both polymerases and their superimposition with TM-Align revealed

221 remarkably similar structures (Supplementary Figure 1) with TM-score of 0.94. This, along 
222 with the characteristic position of the RNA polymerase, initially suggested the inclusion of

223 Andromeda within the phiKMVviruses. Eleven ORFs in Andromeda encode structural

224 proteins including typical gene products observed in members of the Autographivirinae

225 such as tail tubular proteins. The remaining six functionally assigned ORFs encode the

226 terminase small and large subunits as well as the components of the lysis machinery, with

227 the lysozyme belonging to the GH24 family of proteins.

228 With respect to gene regulation in Andromeda, nine $\sigma^{70}$ promoters were predicted, of

229 which three precede the early gene region. This appears to represent a strong signal

230 permitting the rapid transcription of early genes. Due to the presence of an RNA

231 polymerase, we attempted to predict whether phage specific promoters were present. No

232 statistically significant elements were found and of the obtained weak predictions, these

233 were largely found on the non-coding strand. It is possible that Andromeda utilises $\sigma^{70}$

234 elements in the generation of large polycistronic mRNAs. In regard to transcriptional

235 termination, only one rho-independent terminator was found, downstream of gp5. No tRNA

236 genes were discovered in this phage, a feature typical of the Autographivirinae group of

237 phages.

238 Localised single strand interruptions have been reported in phiKMVviruses infecting $P$.

239 aeruginosa [2] and $P$. putida phage tf [31]. A search of the Andromeda genome failed to

240 find the consensus associated with nicks in $P$. aeruginosa phages or $P$. putida phage.

241 Upon subjecting Andromeda genomic DNA to $\mathrm{NaOH}$ denaturation, faint but distinguishable

242 bands were observable on agarose gels which disappear when conducting this reaction

243 after ligation (Supplementary Figure 2). These results prove that localised nicks containing

244 adjacent 5'-phosphate and 3'-hydroxyl groups are present in the Andromeda genome. The

245 low intensity of the bands observed may suggest that only a proportion of phage DNA

246 molecules is nicked. This was previously demonstrated for T5 phage of Escherichia coli

247 where a number of major (present in over $50 \%$ of molecules) and minor (lower presence)

248 canonical nicks are known [32-34].

250 Genetic Heterogeneity in the Andromeda Genome

251 It has previously been discovered that several members of the phiKMVviruses display a

252 high level of genome variability that is localised to specific regions [3]. This is also true in

253 case of Andromeda, which was found to possess the highest number of such variants. In-

254 depth analysis of this genomic feature was conducted in order to gain insights into the

255 nature of the changes and the potential mechanisms at play.

256 Analysis of the deep sequencing data of the Andromeda genome revealed that there are 
at least 80 positions (SNP) where nucleotide substitutions are detected with frequencies ranging from $1 \%$ to $12.48 \%$. Detailed information with respect to each variant is provided in Supplementary Table 2. Cumulative analysis of these frequencies shows that lower frequency variants predominate, with a high level of tapering observed above 3\% (Fig. 4). The mutations are distributed genome-wide, which is dissimilar to the pattern reported previously in other phiKMVviruses where a distinct bias of SNPs distribution (e.g. in genes responsible for phage adsorption) was observed [3]. On a cumulative basis however, it is clear that regions of high variability are observable, which suggests either the consecutive introduction of errors in a single event such as polymerase slippage, or that these represent hotspots for mutation (Fig. 4).

Further analysis of the nature of variants revealed that $73 / 80(91.3 \%)$ lie within coding regions which is unsurprising given that the coding potential of the genome is $95.1 \%$ (Fig. 5). With respect to those variants lying within coding regions, 55 of these result in an amino acid change. Of the 18 silent mutations, 17 were found to exist within the third codon position. This position is overall the predominant location of variants with the second codon position having a similar abundance (Fig. 5). Interestingly, these 17 silent mutations constitute only half of the total variants observed at the third position. The predominance of missense mutations in general is extremely interesting, particularly in the third position. Analysis of the proportion of transversion and transition mutations revealed an unexpected abundance of the former, with $78.75 \%$ of all variants being of this type (including those in non-coding regions). Most transversions are observed in the second codon position, closely followed by the third. In general, whilst there are more potential pathways to transversion mutations, they are in reality less common relative to transitions.

The exchange of a single ring base for a double ring and vice-versa is a significant change and is more likely to result in an alteration at the amino acid level and indeed, one that is less likely to be conservative. The predominance of transversions here explains the high level of non-silent mutations observed across all codon positions and is a unique occurrence not previously reported in other biological systems. The additional abundance of transversion mutations may also further implicate the DNA polymerase as a responsible factor in variant generation through some form of biochemical bias. This is additionally supported by the presence of an insertion mutation in the Andromeda genome within ORF14 (Supplementary Table 2). This variant lies within an 8 base poly-G tract that may have given rise to a slippage event during replication and results in $a+1$ frameshift and truncated ORF14 product.

The possible involvement of the phage DNA polymerases in generation of hypervariability 
292 in genomes of phiKMVviruses was discussed previously [3]. Phage Andromeda's DNA

293 polymerase is similar to those of phiKMVviruses with respect to absence of the

294 thioredoxin-biding domain; however, the genome-wide distribution of variable sites in its

295 genome suggest that different mechanisms of diversity generation might be in play in case

296 of Andromeda.

297

298 Phylogenetic relationships of Andromeda

299 Autographivirinae is a subfamily within Podoviridae, which at the time of writing included

300 several genera, with Teseptimaviruses, Drulisviruses, Friunaviruses, Przondoviruses,

301 Zinderviruses and phiKMVviruses being the largest. Certain features of Andromeda, such

302 as genomic architecture and gene order, presence of nicks and hypervariable sites in its

303 DNA, and the similarity of its gene products such as the RNA/DNA polymerases, tail

304 tubular proteins, major capsid protein, terminase subunits, and lysis machinery, implicates

305 this phage as a member of Autographivirinae, the phiKMVviruses in particular.

306 Nevertheless, it has no detectable nucleotide identity to currently recognised members of

307 phiKMVviruses and has recently been assigned to Bifseptivirus genus within Podoviridae

308 alongside with Pseudomonas phage Bf7 by the International Committee on Taxonomy of

309 Viruses (ICTV proposal 2018.063B.R.Bifseptvirus, approved on 24 February 2019). This

310 highlights the somewhat problematic nature of taxonomic classification of Andromeda and

311 in order to better understand its phylogenetic standing, we conducted an analysis of

312 Andromeda using two conserved proteins - RNA polymerase (RNAP) and terminase large

313 subunit (TerL). BLASTp searches of the NCBI RefSeq database for RNAP and TerL

314 similar to those of Andromeda (see Materials and Methods) resulted in the retrieval of 147

315 complete viral genomes, including 46 recognised by the ICTV (Supplementary Table 1).

316 Almost all the phages used for phylogenetic analyses belong to Autographivirinae (except

317 Pseudomonas phages Andromeda and Bf7 and Aquamicrobium phage P14) and infect a

318 wide range of hosts from Cyanobacteria to Pseudomonas. Reconstruction of phylogenetic

319 trees with IQ-Tree using RNAP and TerL as markers resulted in trees of similar

320 morphology (Fig. 6a and 6b). It can be clearly seen that Teseptimaviruses and

321 Przondoviruses cluster separately from the majority of other phages, while Andromeda

322 belongs to a loose cluster of Autographivirinae phages comprising several smaller genera

323 with only few members (e.g. Bifseptivirus - Pseudomonas phages Andromeda and Bf7,

324 Aqualcavirus - Aquamicrobium phage P14) and unclassified Autographivirinae (e.g.

325 Ralstonia phage RSB3). Although we do not intend to propose any novel classification, we

326 designate this cluster of viruses as "ExophiKMVviruses" for the purposes of discussing this 
327 group and to highlight its distinctive position relative to other Autographivirnae.

328 Despite not currently being classified by ICTV as Autographivirinae, phage Andromeda as

329 well as Bf7 could be included as members of this group based on the presence of a single

330 subunit RNAP in their genomes and their position within the cluster of

331 "ExophiKMVviruses". When we analyse the gene order of representatives of each genus

332 (Fig. 7), we find that the "ExophiKMVviruses", despite being most closely related to

333 Zinderviruses based on sequence identity percent of marker genes, show the downstream

334 localisation of the single-subunit RNA polymerase as is typical for "true" phiKMVviruses.

335 This is a feature conserved across the group (Fig. 7). Analysis of genome size distribution

336 of the putative Autographivirinae (Fig. 8) show that "ExophiKMVviruses" possess a

337 genome size range similar to that of other phiKMVviruses. We have shown previously that

338 phiKMVviruses such as Pseudomonas phage phiNFS lack the thioredoxin binding domain

339 in their DNA polymerases [3] that is responsible for heightened processivity and fidelity of

340 Teseptimaviruses [35, 36]. Molecular modelling and superimposition of the Andromeda

341 and T7 DNA polymerases shows the absence of this domain in Andromeda and highlights

342 another difference between phiKMVviruses and "Exophikmvviruses" in comparison to

343 Teseptimaviruses (Supplementary Figure 3). Taken together, it seems that the

344 "ExophiKMVviruses" might occupy an interface group between the phiKMVviruses and the

345 Teseptimaviruses, although sequence based classification alone being insufficient to

346 adequately resolve the relationships of the Autographivirinae as a whole.

348 Our analysis of the Andromeda phage has once again highlighted the existence of a novel phenomenon within Pseudomonas phages that results in the production of highly heterogeneous populations. Whilst error-prone polymerase activity is almost certainly to be involved in the generation of genomic hypervariability, it unlikely to be the only constituent at play, with intriguing patterns of codon position distribution and localisation suggesting

353 the involvement of some post-replication mechanisms. The taxonomic position of

354 Andromeda suggests that this phenomenon is not unique to phiKMVviruses and could

355 potentially include "Exophikmvviruses" and/or Autographivirinae as a whole. 


\section{Authors and contributors}

358 Conceptualisation: D.J.M. and L.A.K.; data collection and analysis: D.J.M. and T.A.S.;

359 writing - original draft preparation: D.J.M., T.A.S. and L.A.K.; writing - review and editing:

360 T.A.S., D.J.M. and L.A.K

361

362 Funding information

363 This work received no specific grant from any funding agency

364

365 Conflicts of interest

366 The authors declare that there are no conflicts of interest.

367

\section{Ethical statement}

369 No experimental work with humans or animals was performed in this study.

370 


\section{References}

374 1. Kulakov LA, Ksenzenko VN, Kochetkov VV, Mazepa VN, Boronin AM. DNA homology 375 and adsorption specificity of Pseudomonas aeruginosa virulent bacteriophages. MGG Mol Gen Genet 1985; 200: 123-127.

2. Kulakov LA, Ksenzenko VN, Shlyapnikov MG, Kochetkov VV, Del Casale A, Allen CCR, et al. Genomes of "phiKMV-like viruses" of Pseudomonas aeruginosa contain localized single-strand interruptions. Virology 2009; 391: 1-4.

3. Magill DJ, Kucher PA, Krylov VN, Pleteneva EA, Quinn JP, Kulakov LA. Localised genetic heterogeneity provides a novel mode of evolution in dsDNA phages. Sci Rep $2017 ; 7$.

4. Sambrook Joseph, Russell DW. Molecular cloning $\square$ : a laboratory manual. 2001. Cold Spring Harbor Laboratory Press, Cold Spring Harbor, N.Y.

5. Clokie MRJ, Kropinski AM (eds). Bacteriophages: methods and protocols. Volume 1. 2009. Humana Press, New York.

6. Bolger AM, Lohse M, Usadel B. Trimmomatic: a flexible trimmer for Illumina sequence data. Bioinformatics 2014; 30: 2114-2120.

7. Bankevich A, Nurk S, Antipov D, Gurevich AA, Dvorkin M, Kulikov AS, et al. SPAdes:

8. Boetzer M, Henkel CV, Jansen HJ, Butler D, Pirovano W. Scaffolding pre-assembled contigs using SSPACE. Bioinforma Oxf Engl 2011; 27: 578-579.

9. Thorvaldsdóttir H, Robinson JT, Mesirov JP. Integrative Genomics Viewer (IGV): highperformance genomics data visualization and exploration. Brief Bioinform 2013; 14: $178-192$.

10. Carver T, Harris SR, Berriman M, Parkhill J, McQuillan JA. Artemis: an integrated platform for visualization and analysis of high-throughput sequence-based 
experimental data. Bioinformatics 2012; 28: 464-469.

11. Lukashin AV, Borodovsky M. GeneMark.hmm: New solutions for gene finding. Nucleic Acids Res 1998; 26: 1107-1115.

12. Altschul SF, Gish W, Miller W, Myers EW, Lipman DJ. Basic local alignment search tool. J Mol Biol 1990; 215: 403-410.

13. Boratyn GM, Schäffer AA, Agarwala R, Altschul SF, Lipman DJ, Madden TL. Domain enhanced lookup time accelerated BLAST. Biol Direct 2012; 7: 12-12.

14. Jones P, Binns D, Chang H-Y, Fraser M, Li W, McAnulla C, et al. InterProScan 5: genome-scale protein function classification. Bioinforma Oxf Engl 2014; 30: 12361240.

15. Zimmermann L, Stephens A, Nam S-Z, Rau D, Kübler J, Lozajic M, et al. A Completely Reimplemented MPI Bioinformatics Toolkit with a New HHpred Server at its Core. J Mol Biol 2018; 430: 2237-2243.

16. Lowe TM, Eddy SR. tRNAscan-SE: a program for improved detection of transfer RNA genes in genomic sequence. Nucleic Acids Res 1997; 25: 955-964.

17. Laslett D, Canback B. ARAGORN, a program to detect tRNA genes and tmRNA genes in nucleotide sequences. Nucleic Acids Res 2004; 32: 11-16.

18. Naville M, Ghuillot-Gaudeffroy A, Marchais A, Gautheret D. ARNold: A web tool for the prediction of Rho-independent transcription terminators. RNA Biol 2011; 8: 11-13.

19. Zuker M. Mfold web server for nucleic acid folding and hybridization prediction. Nucleic Acids Res 2003; 31: 3406-3415.

20. Bailey TL, Johnson J, Grant CE, Noble WS. The MEME Suite. Nucleic Acids Res 2015; 43: W39-W49.

21. Katoh K, Standley DM. MAFFT Multiple Sequence Alignment Software Version 7: Improvements in Performance and Usability. Mol Biol Evol 2013; 30: 772-780. 
alignment trimming in large-scale phylogenetic analyses. Bioinformatics 2009; 25: 1972-1973.

23. Nguyen L-T, Schmidt HA, von Haeseler A, Minh BQ. IQ-TREE: A Fast and Effective Stochastic Algorithm for Estimating Maximum-Likelihood Phylogenies. Mol Biol Evol 2015; 32: 268-274.

24. Letunic I, Bork P. Interactive Tree Of Life (iTOL) v4: recent updates and new developments. Nucleic Acids Res 2019; 47: W256-W259.

25. Yang J, Yan R, Roy A, Xu D, Poisson J, Zhang Y. The I-TASSER Suite: protein structure and function prediction. Nat Methods 2015; 12: 7-8.

26. Pettersen EF, Goddard TD, Huang CC, Couch GS, Greenblatt DM, Meng EC, et al. UCSF Chimera-A visualization system for exploratory research and analysis. $J$ Comput Chem 2004; 25: 1605-1612.

27. Zhang Y, Skolnick J. TM-align: a protein structure alignment algorithm based on the TM-score. Nucleic Acids Res 2005; 33: 2302-2309.

28. Garneau JR, Depardieu F, Fortier L-C, Bikard D, Monot M. PhageTerm: a tool for fast and accurate determination of phage termini and packaging mechanism using nextgeneration sequencing data. Sci Rep 2017; 7: 8292. by High-Throughput Sequencing. PLOS ONE 2014; 9: e85806.

30. Sajben-Nagy E, Maróti G, Kredics L, Horváth B, Párducz Á, Vágvölgyi C, et al.

31. Glukhov AS, Krutilina AI, Shlyapnikov MG, Severinov K, Lavysh D, Kochetkov VV, et al. Genomic Analysis of Pseudomonas putida Phage tf with Localized Single-Strand DNA Interruptions. PLOS ONE 2012; 7: e51163. 
BACTERIOPHAGE T5+. Proc Natl Acad Sci U S A 1969; 62: 1167-1174.

452 33. Hayward GS, Smith MG. The chromosome of bacteriophage T5: I. Analysis of the

453 single-stranded DNA fragments by agarose gel electrophoresis. J Mol Biol 1972; 63:

454 383-395.

34. Johnston JV, Nichols BP, Donelson JE. Distribution of 'minor' nicks in bacteriophage T5 DNA. J Virol 1977; 22: 510-519.

35. Magill DJ, McGrath JW, O'Flaherty V, Quinn JP, Kulakov LA. Insights into the structural dynamics of the bacteriophage T7 DNA polymerase and its complexes. $J$ Mol Model 2018; 24: 144.

460 36. Tabor S, Huber HE, Richardson CC. Escherichia coli thioredoxin confers processivity 


\section{Legends to Figures}

466

467

468

469

470

471

472

473

474

475

476

477

478

479

480

481

482

483

484

485

486

487

488

489

490

491

492

493

494

495

496

497

498

499

500

501

502

Fig. 1. Electron microscopic image of bacteriophage Andromeda.

Fig. 2. Growth characteristics of bacteriophage Andromeda. A. Determination of adsorption coefficient. B. One-step growth curves: blue line - infected $P$. syringae cells treated with chloroform (intracellular phage); red line - untreated infected $P$. syringae cells (extracellular phage). All experiments were performed in triplicates, error bars represent one standard deviation.

Fig. 3. Genetic organisation of Andromeda and its homology to Pseudomonas bacteriophage Bf7. Areas of substantial similarity between two genomes are shown as trapezia connecting two genome regions coloured according to average nucleotide identity levels of these regions (as calculated by BLASTn). Arrows on the genome map represent identified genes and demonstrate the direction of their transcription. They are coloured to reflect common functions of the encoded products. Putative genes with unidentified function are shown in grey colour. DNAP - DNA polymerase, RNAP - RNA polymerase, MCP - major capsid protein, TerL - terminase large subunit.

Fig. 4. The presence and distribution of variable sites in phage Andromeda. Each dot indicates an SNP, variant frequency is given by its position along the $y$-axis. The histogram on the top shows uneven distribution of variable sites in the phage's genome, the histogram on the right demonstrates the distribution of variant frequencies.

Fig. 5. Summary statistics of SNPs in bacteriophage Andromeda with respect to their position in codons and their effect on amino acids.

Fig. 6. Analysis of phylogenetic relationships of phage Andromeda. A. Consensus phylogenetic tree based on multiple sequence alignment of RNAP amino acid sequences of 147 RefSeq phages; $L G+F+R 6$ substitution model. B. Consensus phylogenetic tree based on multiple sequence alignment of TerL amino acid sequences of 147 RefSeq phages; LG+R5 substitution model. Consensus trees were constructed from 1000 bootstrap trees, numbers in parentheses are bootstrap supports (\%). Both trees were rooted with a cluster of 11 cyanophages as outgroup. "Exophikmvviruses" are outlined by a red box.

Fig. 7. Comparison of positions of RNA (blue) and DNA (red) polymerases in representatives of different genera. In Zinderviruses (SP6), Teseptimaviruses (T7) and Przondoviruses (K11) DNA polymerase is adjacent to structural genes and is positioned downstream of RNA polymerase, while for the rest of the viruses the opposite is true. 
bioRxiv preprint doi: https://doi.org/10.1101/619015; this version posted March 5, 2020. The copyright holder for this preprint (which was not certified by peer review) is the author/funder, who has granted bioRxiv a license to display the preprint in perpetuity. It is made available under aCC-BY-NC-ND 4.0 International license.

503

504 Fig. 8. Genome size distribution among viruses of different taxonomic groups analysed in this 505 study.

506 


\section{Legends to Supplementary Data}

508

509 Supplementary Figure 1. Superimposition of three-dimensional structures of RNA polymerases

510 from Pseudomonas phages Andromeda (salmon) and phiNFS (purple).

512 Supplementary Figure 2. Visualisation of the localised single stranded interruptions in the

513 Andromeda's genome. (A) GeneRuler 1 kb Plus DNA Ladder ladder; (B) Untreated phage DNA;

514 (C) $\mathrm{NaOH}$ treated DNA, major DNA hydrolysis products are labelled (1-3); (D) $\mathrm{NaOH}$ treated DNA 515 which was preliminarily ligated by T4 DNA ligase.

516

517 Supplementary Figure 3. Superimposition of three-dimensional structures of DNA polymerases

518 from Pseudomonas phage Andromeda (salmon) and Enterobacteria phage T7 (blue, model

519 derived from PDB 1SKR).

520

521 Supplementary Table 1. NCBI RefSeq phage genomes used in this study.

522

523 Supplementary Table 2. Detailed description of SNPs and indels in phage Andromeda genome.

524

525 Supplementary File 1. Phage Andromeda annotated genome (Genbank format). 
bioRxiv preprint doi: https://doi.org/10.1101/619015; this version posted March 5, 2020. The copyright holder for this preprint (which was not certified by peer review) is the author/funder, who has granted bioRxiv a license to display the preprint in perpetuity. It is made available under aCC-BY-NC-ND 4.0 International license. 


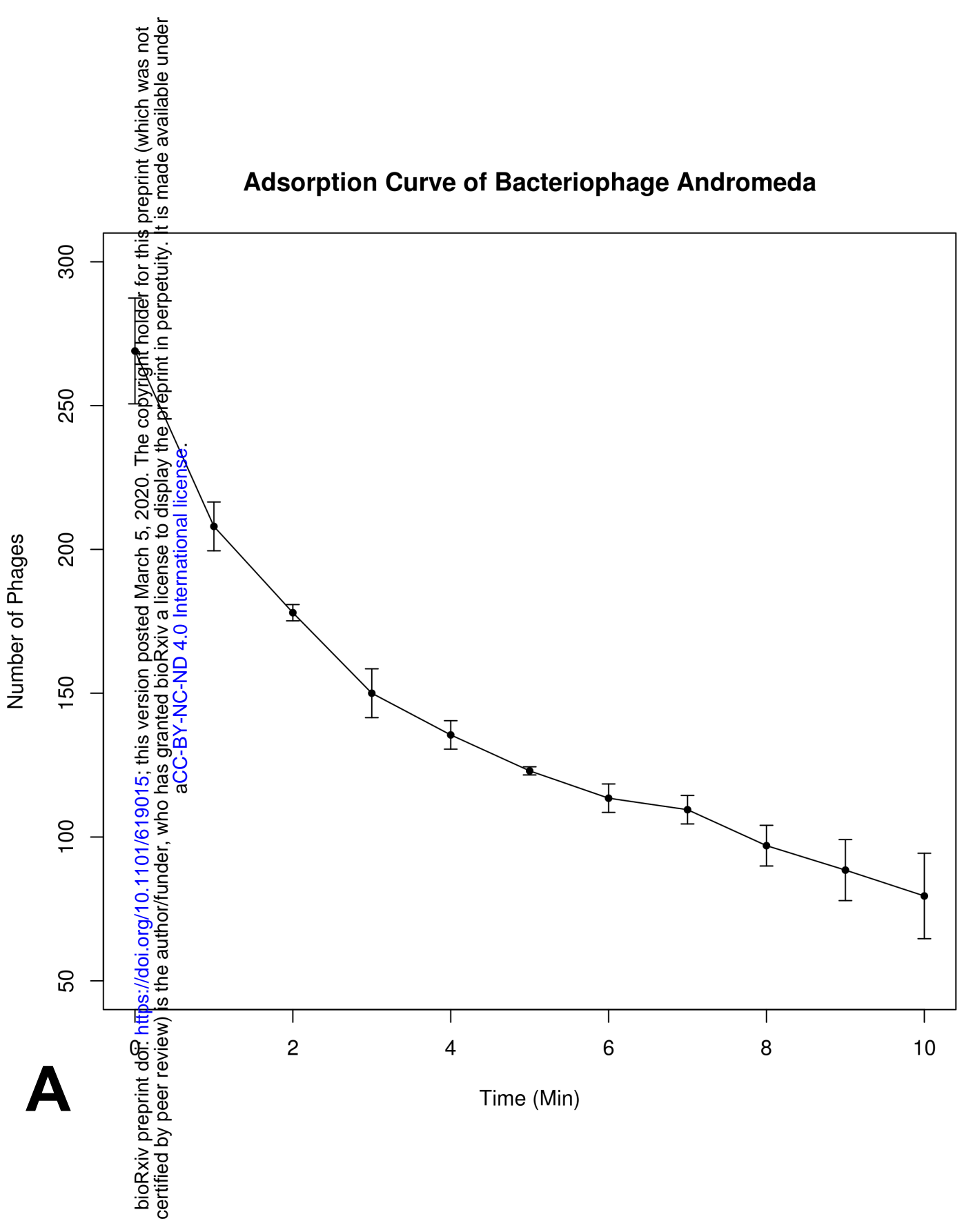

One Step Growth of Bacteriophage Andromeda

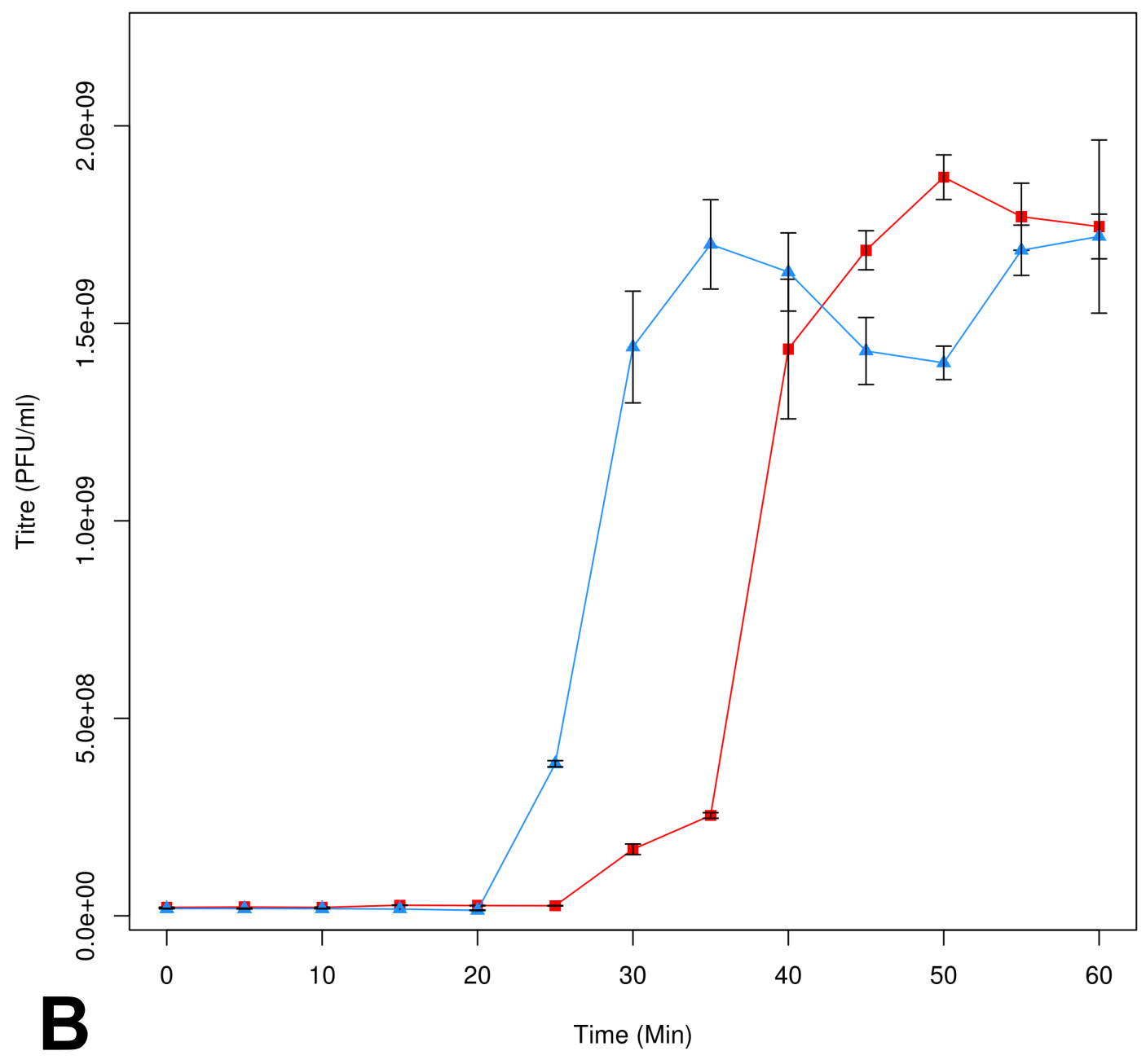




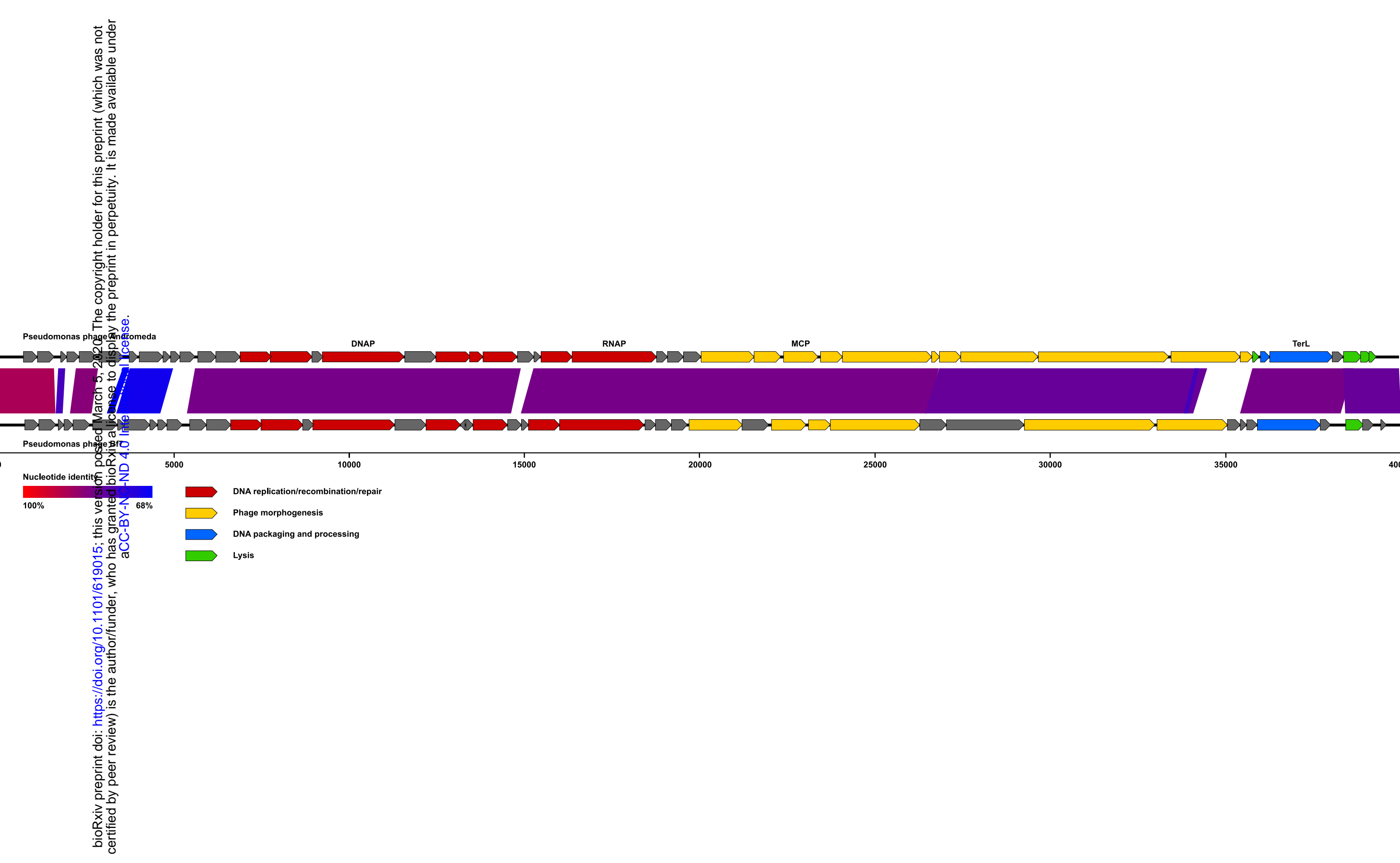




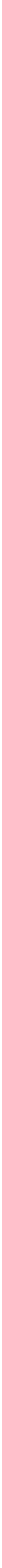


Bacteriophage Andromeda SNP Summary Statistics

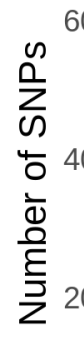
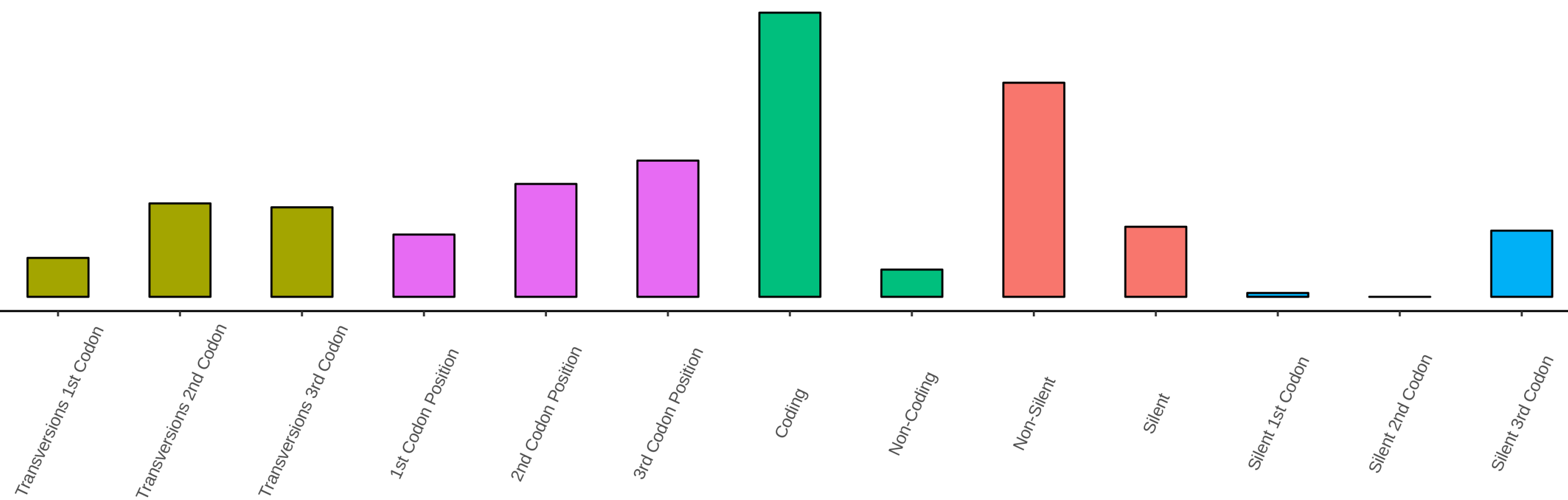

कิ
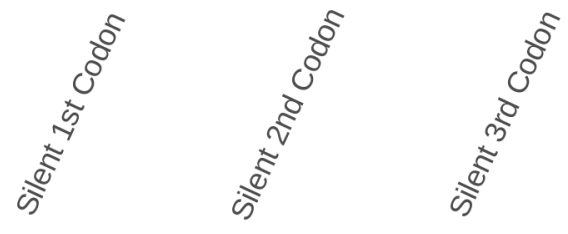


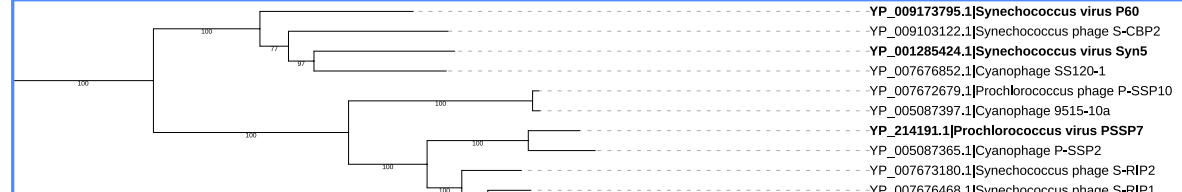

outgroup

bioRxiv preprint doi: https://doi.org/10.1101/619015; this version posted March 5, 2020. certified by peer review) is the author/funder, who has granted bioRxiv a license to display-therepreprimpinf perpetuity. It is made available under

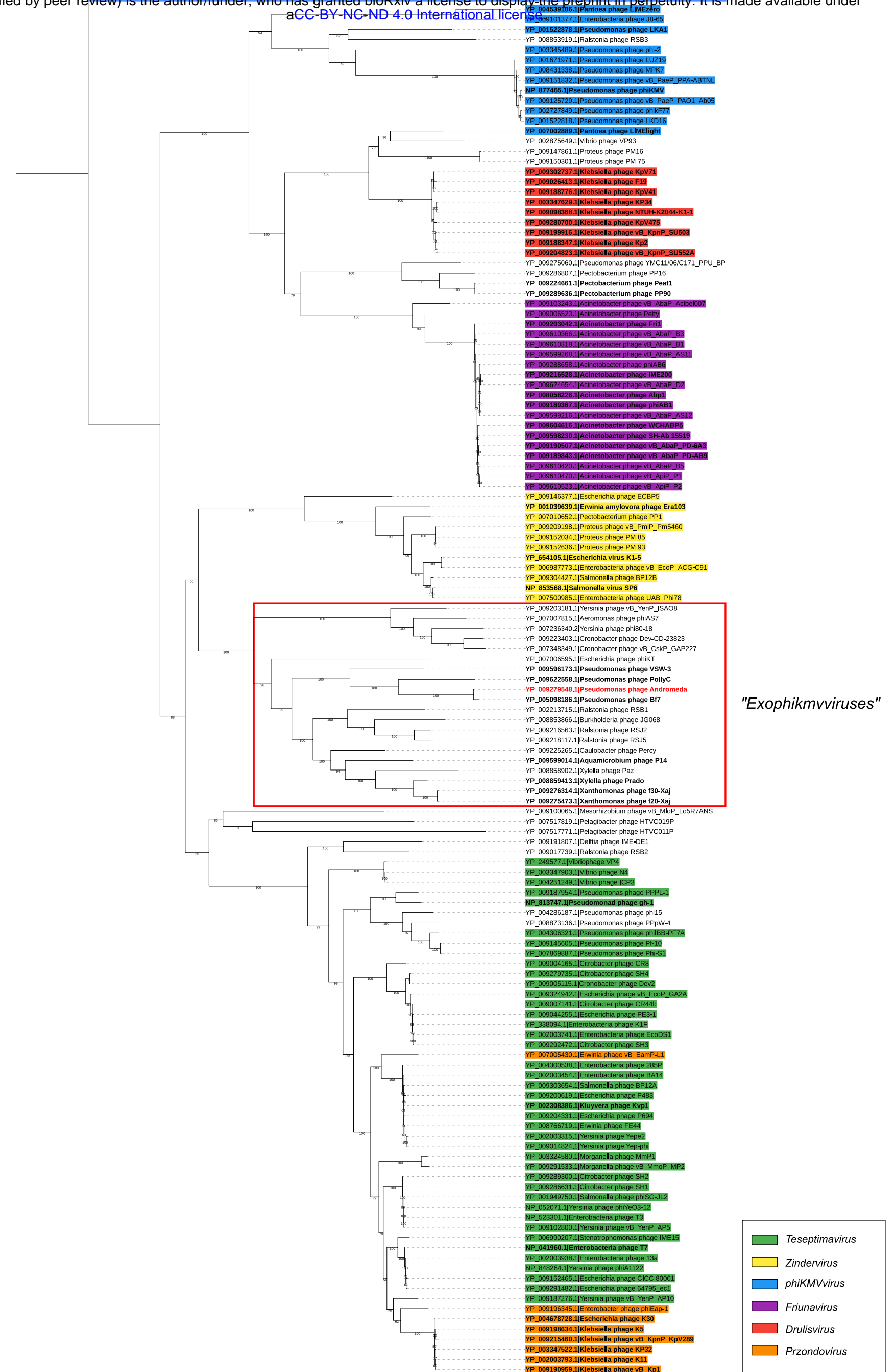




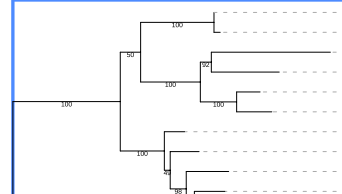

YP_007672692.1||Prochlorococcus phage
YP_005087411.1|Cyanophage 9515-10

YP 009103160.11Synechococcus p p

YP_007676860.11Cyanophage SS120-1

YP_009173820.1|Synechococcus virus P60

YP_001285469.1Synechococcus virus Syn5

YP-0091038915.1Synnechocococcus phage S-CBP1

outgroup

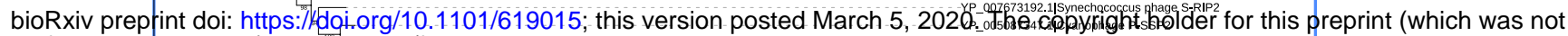

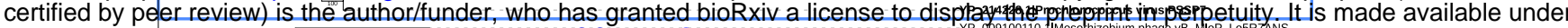

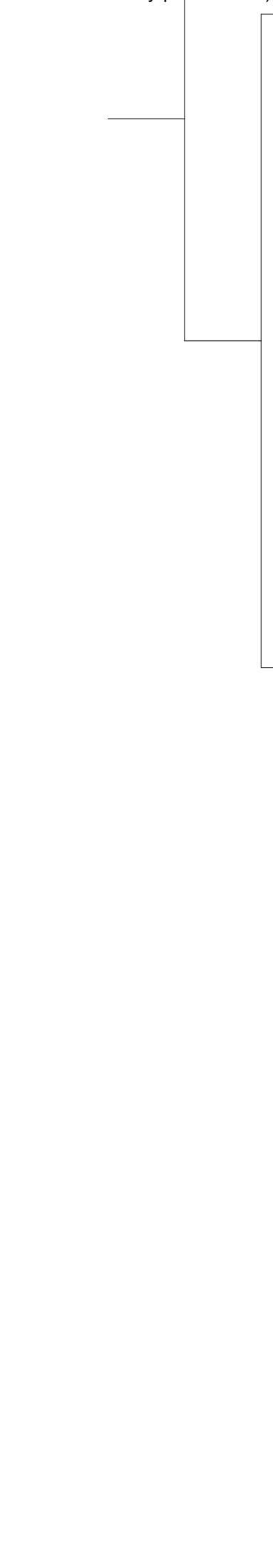

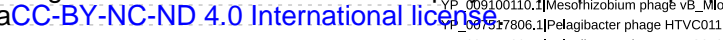

YP_007517865.1Pelagibacter phage HTVC01P

YP_009191792.1|Delttia phage IME-DE

YP. 004251296.1 Vibrio phage ICP

YP 003347946.1|Vibrio phage N4

NP_813786.1Pseudomonad phage gh-1

TP_009187993.1Pseudomonas phage PPPL-1

YP 008873175 1Pseudomanas phage PPpw

YP 007869923 .

YP_009145642.1|Pseudomonas phage Pf-10

YP_004306358.1Pseudomonas phage philBB-PF7

YP. 009004210.1 Citrobacter phage CR8

YP_0092797/5.1CCirobacter phage SH4

YP 009292513 1Citrobacter phage Dev2

YP 009007190.11Citrobacter phage CR44

YP_009324988.1Escherichia phage VB_EcoP_GA2A

YP 338131.1|Enterobacteria phage K1F

YP_002003787.11Enterobacteria phage EcoDS

YP_009044295.1Escherichia phage PE3-11

YP_009201567.11 Morganella phage vB_Mm

YP_006990246.11Stenotrophomonas phage IME15

YP_009152457.1|Escherichia phage CICC 800

NP_848309.1|Yersinia phage phiA1122

YP_ 002003984.1|Enterobacteria phage 13a

P__009291523.1|Escherichia phage 64795_ec1

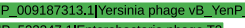

YP 009102839 . IYersinia phage vB Y Y

NP_052122.1|Yersinia phage phiYeo3-12

YP_009286675.1|Citrobacter phage SH1

YP_001949795.1Salmonella phage phisG- J

YP 000289344.1C Citrobacter phage SH2

PP_009196384.1|Enterobacter phage phiEap-1

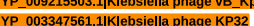

YP 009190952 2 1Klebsiella phage VB KP1

YP_004678767.1|Escherichia phage K30

YP_009198673.1|Klebsiella phage K5

YP_002003836.1|Klebsiella phage K11

YP_007005472.1|Eminia phage vB_EamP-L1

YP_002003498.11Enterobacteria phage BA

YP 002308426 1 1Kluyvera phage KVy

YP_008766763.11Ewinia phage FE44

YP_002003357.1/Yersinia phage Yepe

YP_009014864.1/Yersinia phage Yep-ph

YP_004300581.1Enterobacteria phage 285

YP_009200625.1Escherichia phage P483

YP_009146419.1E Escherichia phage ECBP5

YP 001039677.11E Erwinia amylovora phage Era103

YP_ 006987808.11Enterobacteria phage vB ECOP ACG-C91

YP_654141.1|Escherichia virus K1-5

YP_007010685.1Pectobacterium phage $P$ P

YP_009152659.1|Proteus phage PM 93

YP_009209235.1Proteus phage VB_PmiP_PPm546

NP 853601.1.1Salmonella virus SP6

Y__007501024.11Enterobacteria phage UAB_Phi78

YP_009304461.1|Salmonella phage BP12B

YP_009622574.1|Pseudomonas phage PollyC

YP_009279565.1|Pseudomonas phage Andro

(P.

YP_002213730.1|Ralstonia phage RSB1

YP 009216578.1Ralstonia phage RS12

YP_009218130.1/Ralstonia phage RSJ5

YP_007006609.1|Escherichia phage phikT

YP_009203198.1YYersinia phage vB_YenP_ISACA

YP_007007799.1/Aeromonas phage phiAS7

YP_-007230350.2YYersina phage phiro-18

YP_009223418.1||Cronobacter phage Dev-CD-23823

YP_009225282.11Caulobacter phage Percy

YP_009599029.11Aquamicrobium phage $P$

YP_008858920.1|Xylella phage Paz

YP_008859432.1Xylella phage Prado

YP_-00922

YP_009276349.1|Xanthomonas phage $130-X$

YP_.009101391.1| Enterobacteria phage J8-65

YP_ 004539123.1|Pantoea phage LIMEzer

P_.003345504.11Pseudomonas phage phi-2

PP_002727866.1PPseudomonas phage phikF7

P 001522835.1Pseudomonas phage LKD16

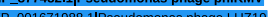

aeP_PPA-ABTNL

nonas phage MPK

YP_009125746.1|Pseudomonas phage VB_PaeP_PAO1_Ab05

PP_009286821.1Pectobacterium phage PP16

PP_009289655.1|Pectobacterium phage PP9

YP _009147874 1Proteus phage PM16

YP 009150311.1|Proteus phage PM 75

P__002875662.1|VVibrio phage VP93

YP_007002903.1||Pantoea phage LIMElig

YP_003347645.1|Klebsiella phage KP34

P_009188791.1|Klebsiella phage KpV41

YP_009204837.1|Klebsiella phage vE

YP_009199931.1| KKlebsiella phage UB KpnP SU1509

YP_009280714.1|Klebsiella phage KpV475

YP_009188361.1|Klebsiella phage Kp2

P__009302751.1|Klebsiella phage KpV7:

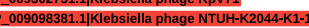

YP. 009103262.1/Acinetobacter phage vB AbaP.Acibel007

YP_009189826.1|Acinetobacter phage VB_AbaP_PD

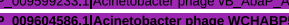

008058244.11 Acinetobacter phage AbD1

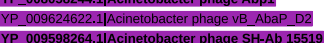

095505204.1Aacinetovacter phage SH-Ab 15

P . 009610437.1Acinetobacter phage VB Aba

YP_009189384.1|Acinetobacter phage phiAB

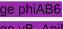

YP_009190477.1|Acinetobacter phage VB_AbaP.PD- 
Andromeda

$\square D D D \square D \square D O D D \square \square \square$

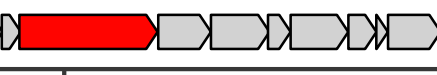

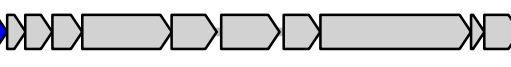

20000

$>\square D D D \longrightarrow D D C D$

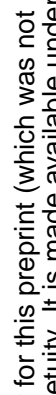

PollyC

10000

$\sqrt{300}$

$30000 \quad 40000$

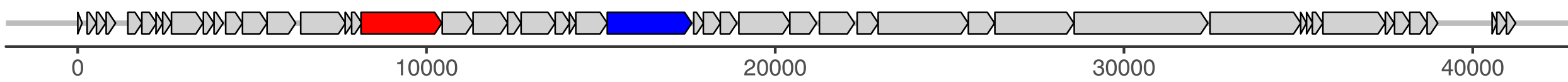

VSW-3

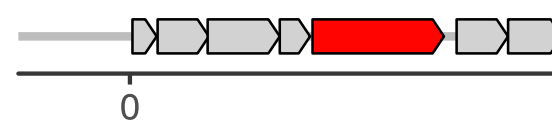

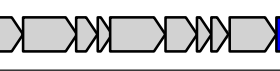

$\frac{D D D}{20000}$

10000

$102+200$

DDODOODDDODDD

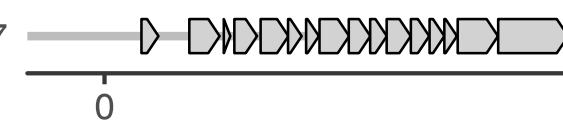

$>\square \square \square \times C D \square \times 20 x$

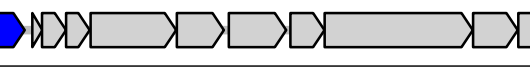

30000

40000

RSJ5

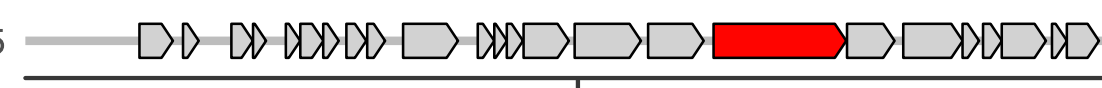

20000

30000

$\frac{D X \square}{40000}$ 10000

20000

$\square D \square \times \square \square \square$

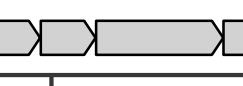
30000

$>D D D D$

Prado

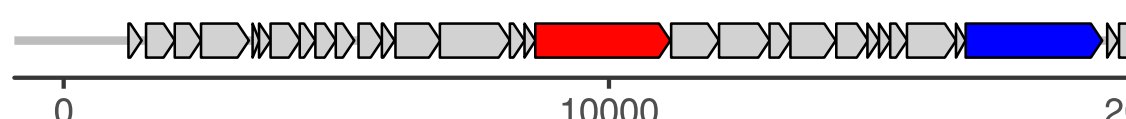

$D D D \square \times \square D \square \square \square \times$

20000

(3000

P14

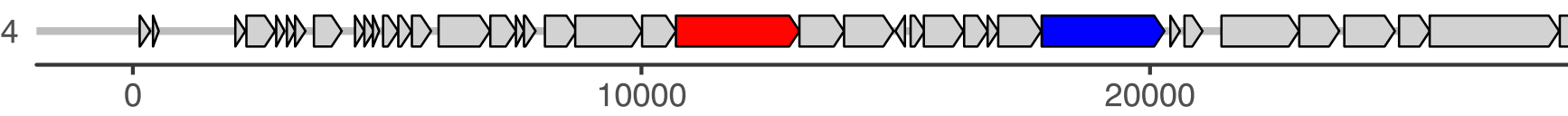

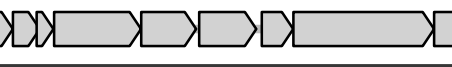

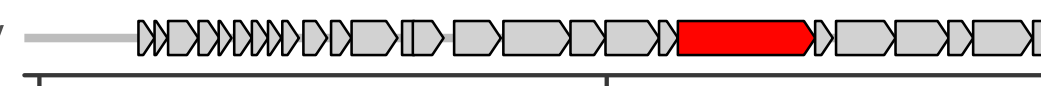

10000

20000

30000

30000

30000

WDO 202002 40000

KP34

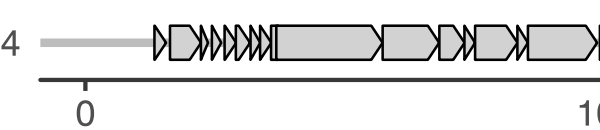
10000

$D D \square D D \square D O D D \square X D D$ 20000

$x \longdiv { 2 }$

$\lambda$

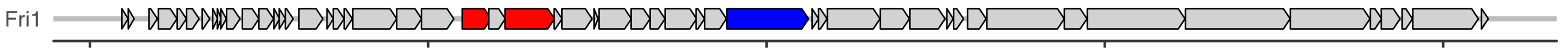
\begin{tabular}{llll}
\hline & 10000 & 20000 & 30000
\end{tabular}

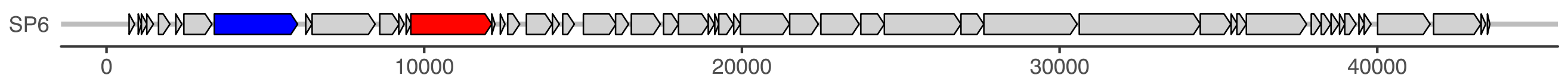

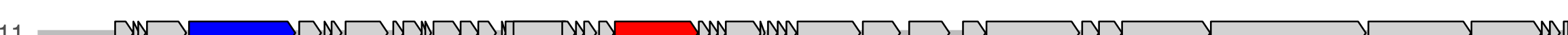

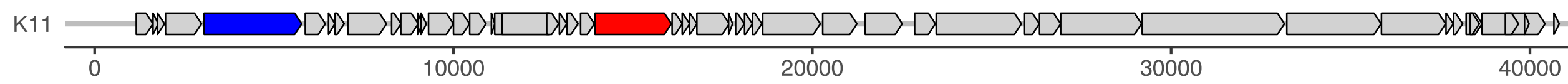

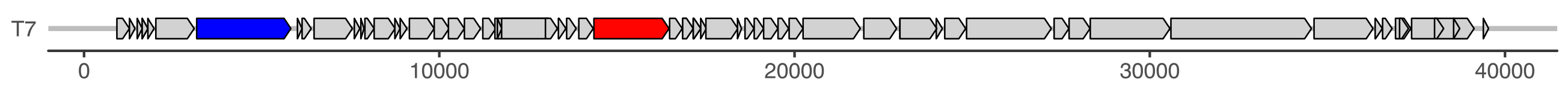

$20000-30000-40000$


Phage genome length distribution

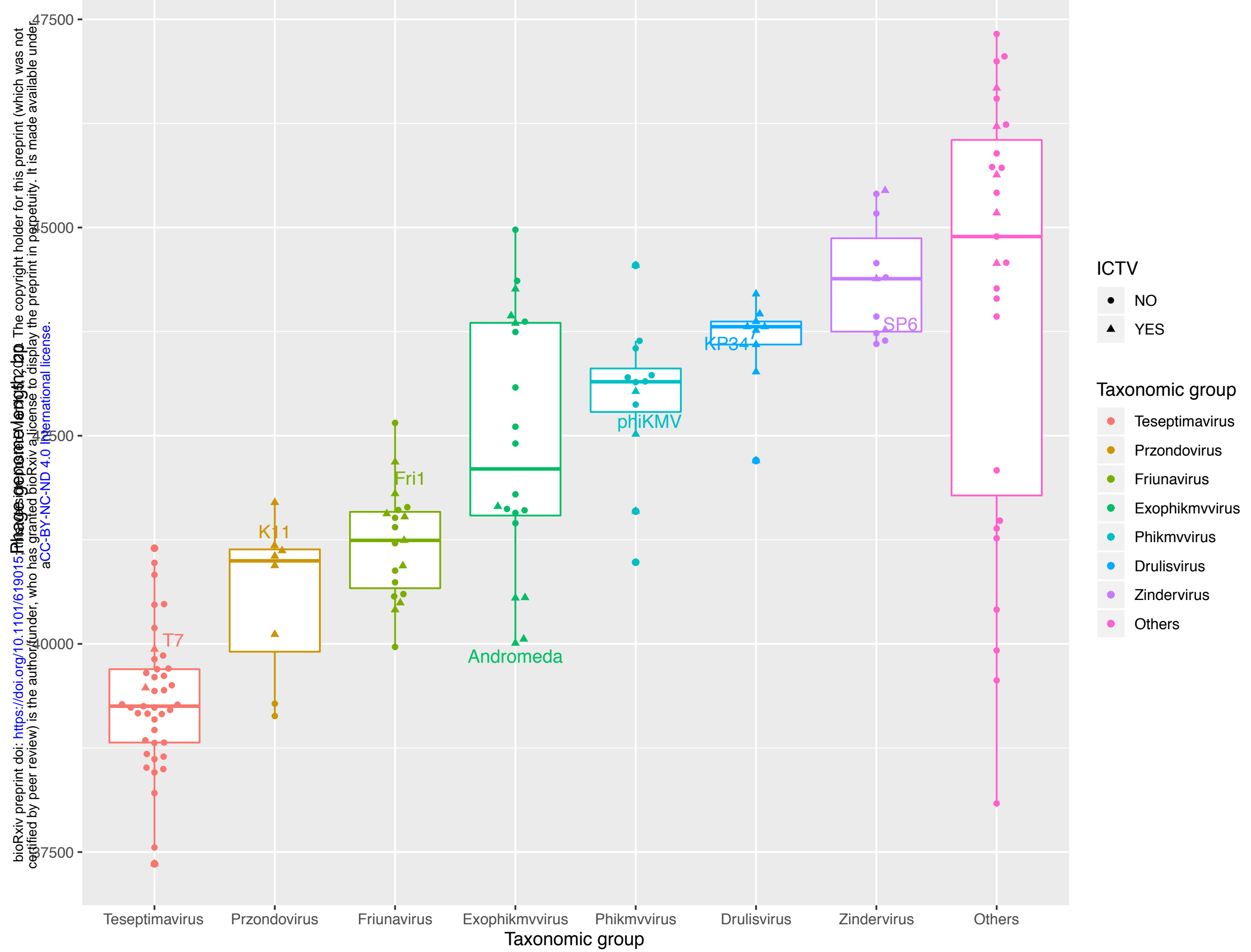

